\title{
Sustained Effects of Once-Daily Memantine Treatment on Cognition and Functional Communication Skills in Patients with Moderate to Severe Alzheimer's Disease: Results of a 16-Week Open-Label Trial
}

\author{
Jörg B. Schulz ${ }^{\mathrm{a}, *}$, Michael Rainer ${ }^{\mathrm{b}}$, Hans-Hermann Klünemann ${ }^{\mathrm{c}}$, Alexander Kurz ${ }^{\mathrm{d}}$, Stefanie Wolf ${ }^{\mathrm{a}, \mathrm{e}}$, \\ Kati Sternberg ${ }^{\mathrm{f}}$ and Frank Tennigkeit ${ }^{\mathrm{f}}$ \\ ${ }^{a}$ Department of Neurology, University Medical Center, RWTH Aachen, Aachen, Germany \\ ${ }^{\mathrm{b}}$ Memory-Clinic, Department of Psychiatry, Donauspital SMZ Ost, Vienna, Austria \\ ${ }^{\mathrm{c}}$ Department of Psychiatry, University of Regensburg, Regensburg, Germany \\ ${ }^{\mathrm{d}}$ Department of Psychiatry and Psychotherapy, Klinikum rechts der Isar, Technische \\ Universität München, München, Germany \\ ${ }^{\mathrm{e}}$ Clinic of Psychiatry and Psychotherapy, University of Bonn, Bonn, Germany \\ ${ }^{\mathrm{f}}$ Central Medical Affairs, Merz Pharmaceuticals GmbH, Frankfurt/Main, Germany
}

Accepted 19 February 2011

\begin{abstract}
The present study evaluated the effects of once-daily memantine $(20 \mathrm{mg})$ treatment on cognition and communication in patients with moderate to severe Alzheimer's disease (AD). In a multicenter, single-arm open-label study, outpatients diagnosed with $\mathrm{AD}$ (MMSE <20; $n=97$ ) were titrated from $5 \mathrm{mg}$ to $20 \mathrm{mg}$ once-daily memantine over 4 weeks. Once-daily memantine treatment $(20 \mathrm{mg})$ was then continued for 8 weeks, followed by a 4-week wash-out period. The primary efficacy endpoint was the change from baseline in the Consortium to Establish a Registry for Alzheimer's Disease -Neuropsychological Battery (CERAD-NP) total score. Secondary efficacy endpoints included change from baseline in Functional Communication Language Inventory (FLCI) and ADCS-ADL 19 total score, and the response from baseline in Clinical Global Impression of Change (CGI-C). The CERAD-NP total score improved significantly after 12 weeks of once-daily memantine treatment compared with baseline $(5.9 \pm 8.8 ; p<0.0001)$. The FLCI total score improved significantly after 12 weeks compared with baseline $(4.4 \pm 6.8$; $p<0.0001$ ). These significant improvements were already observed after 4 and 8 weeks of once-daily memantine treatment and persisted after a 4-week wash-out period. ADCS-ADL 19 total scores showed only slight increases from baseline, and CGI-C indicated that the majority of patients experienced an improvement or stabilization of the disease after 12 weeks. At least one Treatment-Emergent Adverse Event was reported by 38 (39.2\%) patients. In patients with moderate to severe AD, once-daily memantine $(20 \mathrm{mg})$ treatment significantly improved cognition and functional communication and was found to have a favorable safety and tolerability profile.
\end{abstract}

Keywords: Alzheimer's disease, clinical trial, cognition, functional communication, memantine, once-daily, open-label

\footnotetext{
${ }^{*}$ Correspondence to: Jörg B. Schulz, Department of Neurology, University Medical Center, RWTH Aachen, Pauwelsstr. 30, 52074 Aachen, Germany. Tel: +49 241808 9600; Fax: +49 241808 2582; E-mail: jschulz@ukaachen.de.
} 


\section{INTRODUCTION}

Alzheimer's disease (AD) is a progressive neurodegenerative disease characterized by cognitive impairment, a gradual decline in ability to perform daily functions, and changes in behavior that ultimately result in the complete dependence of a patient on their caregiver [1, 2]. Besides cognitive, functional, and behavioral problems, impairment in functional communication - the pragmatic use of language and expressiveness in everyday life - is another key symptom associated with $\mathrm{AD}$ and one which is currently underestimated in patients with AD. Patients with impaired functional communication have difficulties with discourse, word-finding ability (anomia), and comprehension. As functional communication is a major part of social life, impairments in the ability to communicate have a great impact on the everyday life of both patients and caregivers. With the progression of $\mathrm{AD}$, patients find it increasingly difficult to communicate and become more dependent, isolated, frustrated, aggressive, and fearful. Consequently, caregivers find interaction with the patient difficult or impossible, and they may be confronted with aggressive behavior, making the task of caring for the patient more difficult. Additionally, there may be long-term repercussions of impaired communication, such as family disruption and institutionalization of the patient. A survey of dementia caregivers in Europe, reported that $88 \%$ of caregivers stated problems with communication as an area of difficulty when caring for patients with $\mathrm{AD} ; 36 \%$ of caregivers stated that difficulty with communication was the most problematic symptom [3].

Therapies that effectively treat functional communication impairments in patients with AD may enable patients to retain their ability to express their needs. This could, for example, ensure adequate palliative care in areas such as pain management. However, as functional communication is an emerging field of investigation, data concerning the prevalence and incidence of impaired functional communication is scarce. So far, only one study has explicitly reported on speech after treatment with anti-dementia drugs [4]. Furthermore, there are few validated scales for functional communication in $\mathrm{AD}$ that may be used to monitor the effectiveness of AD therapy.

We therefore evaluated once-daily memantine treatment on AD-related cognition and communication problems in patients with moderate to severe AD. Furthermore, we evaluated, whether the FLCI is a sensitive instrument to evaluate changes in language and speech.
The FLCI was chosen, because patients with moderate to severe AD are still able to perform its items.

\section{MATERIALS AND METHODS}

The overall objective of the study was to evaluate the effects of once-daily memantine treatment on cognitive function and communication in patients with dementia of Alzheimer's type and a Mini Mental State Examination (MMSE) $<20$ at screening. In addition, the safety and tolerability of once-daily memantine was assessed. The study was prospective, single-arm, multi-center, and open-label.

\section{Patients}

Patients were screened and treated at 21 study centers in Germany and Austria. The study was approved by an Independent Ethics Committee (IEC) and was performed in accordance with the International Conference on Harmonisation (ICH) guideline for Good Clinical Practice (GCP), the ethical principles of the Declaration of Helsinki, and the national regulations. All patients provided written informed consent to participate.

Patients eligible for study inclusion were male or female outpatients aged $\geq 50$ years, with a current diagnosis of probable $\mathrm{AD}$ according to the National Institute of Neurological and Communicative Diseases and Stroke/Alzheimer's Disease and Related Disorders Association (NINCDS-ADRDA), and the Diagnostic and Statistical Manual of Mental Disorders Text Revision (DSM-IV-TR) criteria for dementia of the Alzheimer's type, and consistent with results of magnetic resonance imaging (MRI) or computer tomography (CT) scans. The MMSE total score was $<20$ at screening. In addition, eligible patients had $\geq 8$ years of education, were experiencing communication difficulties assessed by Clinical Global Impression (CGI) and judged by a physician to be consistent with a diagnosis of $\mathrm{AD}$, and had sufficient sight and hearing to undertake the study-related procedures and psychometric tests. Exclusion criteria included evidence of any clinically significant and active central nervous system disease (other than AD) or disease of any other major body system, unstable psychiatric illness (other than symptoms associated with AD), including psychotic disorders and bipolar or unipolar depression, or a modified Hachinski Ischemia score $>4$.

Patients being treated with an acetylcholinesterase inhibitor (AChEI) were included if the dose was sta- 
ble during the last 3 months prior to study entry. Dose changes, switching from one AChEI to another, or discontinuation of the AChEI treatment during the study period was prohibited.

\section{Study procedures}

Patients were screened for eligibility one week prior to baseline (Fig. 1). Eligible patients meeting all inclusion criteria and who did not meet any of the exclusion criteria were enrolled by the study investigators to receive treatment with once-daily memantine. As this was an open-label, single-arm study, all patients received the same treatment. Once-daily memantine was titrated upwards from $5 \mathrm{mg}$ to $20 \mathrm{mg}$ over 4 weeks (Weeks 1 to 4): $5 \mathrm{mg}$ in Week $1,10 \mathrm{mg}$ in Week 2, $15 \mathrm{mg}$ in Week 3 and $20 \mathrm{mg}$ in Week 4 (Fig. 1). From Weeks 5 to 12 (8 weeks), patients received a $20 \mathrm{mg}$ maintenance dose of once-daily memantine. This was followed by a wash-out period of 4 weeks (Weeks 13 to 16) (Fig. 1). Patients were instructed to take study medication orally with water and were informed that it could also be taken with a meal.

The study period was 17 weeks in total (including the one-week screening period), which included 12 weeks of treatment with once-daily memantine.

\section{Study endpoints}

The primary efficacy endpoint was the mean change from baseline to Week 12 in the Consortium to Establish a Registry for Alzheimer's DiseaseNeuropsychological Battery (CERAD-NP) total score $[5,6]$. Secondary efficacy endpoints included the mean change from baseline to Week 4, Week 8, Week 12, and from Week 12 to Week 16 in the CERAD-NP total score and the single-item subscores of the CERAD-NP sub-tests (see 'Assessment scales' section below), the

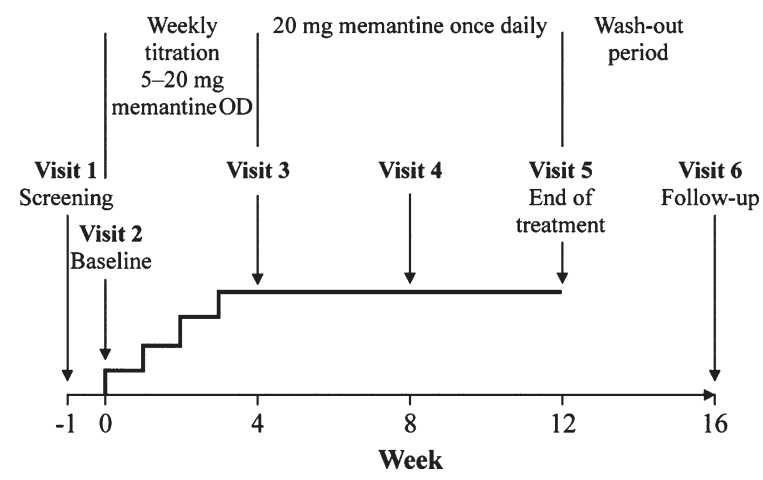

Fig. 1. Study design.
FLCI total score [7], the 19-item Alzheimer's Disease Cooperative Study-Activities of Daily Living Inventory $\left(\mathrm{ADCS}_{-} \mathrm{ADL}_{19}\right)$ total score $[8,9]$, the Clinical Global Impression of Change (CGI-C) [10], and in the Phonemic Verbal Fluency Test (S-words) [11].

Assessments for the primary efficacy endpoint (CERAD-NP total score) were performed at every visit from baseline to Week 16. All secondary efficacy variables were assessed at every visit from baseline to Week 16, with the exception of the CERAD-NP single-item subscore for MMSE which was assessed at screening and at each visit from Week 4 to Week 16, and CGI-C, which was assessed at each visit from Week 4 to Week 16.

Safety and tolerability assessments included the recording of adverse events (AEs), vital signs and abnormalities in laboratory evaluations (hematology, biochemistry, and urinalysis). AEs were evaluated for severity, seriousness, and causal relationship with the study drug. Serious AEs (SAEs) were defined as AEs that resulted in death, were life-threatening/disabling, or required hospitalization. AEs and vital signs were assessed at each visit from screening to Week 16. Laboratory evaluations were conducted at screening and Week 16.

\section{Assessment scales}

The CERAD-NP test battery is a standard measure of cognition in patients with dementia and assesses language, learning, construction, and memory. The test battery is composed of 5 sub-test groups derived from previously-established cognitive tests: verbal fluency (animal naming), Modified Boston Naming Test (MBNT), MMSE, constructional ability (constructional praxis, figures recall), word list memory (word list learning, word list recall, word list recognitiondiscriminability) $[5 ; 6]$. The CERAD-NP total score is the sum of the single-item subscores of the sub-tests (excluding MMSE and figures recall) with scores in the range of 0-100 (high numbers reflect good cognitive status).

The FLCI test battery is a standardized instrument used to evaluate functional communication abilities in moderate to severe dementia patients [7]. Ten subtests assess greeting and naming, writing, following commands, gesture, sign comprehension and objectpicture matching, word reading and comprehension, answering questions, reminiscing, pantomime, and conversation. The FLCI total score is the sum of the ten sub-test scores with a range of 0-87 (high numbers reflect good functional communication) [7]. In the 
present study, for the first time, a German translation of the inventory was used.

The ADCS-ADL 19 scale is a comprehensive battery of ADL questions used to measure the day-to-day functional capabilities of patients with moderate to severe dementia. A subset of 19 items rate the patient's ability to eat, dress, bathe, telephone, travel, shop, and perform other household chores. The ADCS-ADL 19 total score is the sum of the 19 subscores with a range of 0-54 (low scores indicate functional impairment) $[8,9]$.

The CGI-C is a scale to assess the overall treatment response in patients with $\mathrm{AD}$. The clinician rates the improvement or deterioration of a patient's illness as compared with the baseline state, using a 7-point rating scale where $1=$ very much improved and $7=$ very much worse [10].

The Phonemic Verbal Fluency Test (S-words) is used to measure the letter verbal fluency in patients with AD. Subjects are instructed to say as many words as possible beginning with the letter ' $S$ ' in a period of one minute [11].

\section{Statistical analyses}

Data analysis was performed on three sets of patient samples - the safety evaluation set (SES) (all enrolled patients who received study medication at least once), the full analysis set (FAS) (all enrolled patients from the SES who had a baseline CERAD-NP total score, reached the maximum once-daily memantine dose of $20 \mathrm{mg}$ after the 4 -week titration period and had $\geq 1$ post-baseline CERAD-NP total score measurement at Week 4, Week 8, or Week 12), and the per protocol set (PPS) (all subjects from the FAS for whom no major protocol deviations were reported). The evaluation of safety was based on the SES. In the evaluation of effects, PPS was used for confirmatory analyses. Significant effects were concluded if the statistical analyses based on both FAS and PPS data sets showed statistically significant results.

Sample size was calculated based on a 4-point change from baseline to Week 12 in CERAD-NP total score, with a standard deviation of 11.5 points. On this basis, a sample of 89 evaluable patients assured a statistical power of $90 \%$, using a one-sided, single-group, $t$-test at the $2.5 \%$ level of significance. However, to account for the possibility that $25 \%$ of patients from the SES might not be included in the final analysis (due to a missing baseline CERAD-NP total score, failure to reach the maximum once-daily memantine dose of $20 \mathrm{mg}$ after a 4-week titration period, no post-baseline CERAD-NP total score observation, or a major proto- col deviation), enrolment of 119 subjects in total was required to achieve 89 patients for evaluation.

Where data was missing for CERAD-NP total scores at Week 12 (due to missing subscores), an imputation strategy was applied to each missing subscore. The last observation carried forward approach was used if the last available post-baseline subscore at Week 4 or Week 8 showed an improvement over baseline (or screening for MMSE), or no change. If there was deterioration at the last available post-baseline subscore at Week 4 or Week 8 compared with baseline (or screening for MMSE), then a worst-case imputation was implemented, i.e., the missing CERAD-NP subscore at Week 12 was set to 0 . The CERAD-NP total score for Week 12 was then calculated after imputation of the missing subscores. The same rule applied to data missing for CERAD-NP subscores at Week 4 and Week 8. However, if no previous post-baseline subscore was available, then the next post-baseline subscore was considered (with the exception of Week 16, due to treatment being stopped at Week 12). In the case of an improvement over baseline (or screening for MMSE) or no change, the missing subscore was imputed by the baseline outcome (or screening outcome for MMSE). If there was a deterioration compared with baseline (or screening for MMSE), the subscore was to be carried backwards to impute the missing information. CERAD-NP total scores at Week 4 and Week 8 were then calculated after imputation of the missing subscores. Missing CERAD-NP subscores at Week 16 were not imputed and, consequently, respective CERAD-NP total scores at Week 16 could not be calculated. Imputation of missing data for FLCI, ADCS-ADL 19 , and the Phonemic Verbal Fluency Test was applied, similar to the methodology for missing CERAD-NP scores, except that if there was no postbaseline measurement at Weeks 4,8 , or 12 , the patient was excluded from the statistical analysis. Missing values for CGI-C were not imputed and, in these instances, patients were removed from the statistical analysis.

Analysis of the primary and secondary efficacy endpoints was performed on the FAS and the PPS using a one-sided, single-group, $t$-test at a significance level of $2.5 \%$. Two-sided confidence intervals (CI) at a $95 \%$ confidence level for the mean change of patient status compared with the baseline situation were calculated (summary statistics of the secondary efficacy endpoints were predefined; calculation of confidence intervals and $p$-values was conducted post-hoc). A post-hoc subgroup analysis defined by MMSE score at screening (MMSE < 15 and MMSE $\geq 15$ ) was performed on the FAS for the primary efficacy variable, CERAD-NP 
total score. Safety and tolerability analyses were conducted on the SES. Descriptive statistics of AEs were calculated for treatment-emergent AEs (TEAEs) only. TEAEs were defined as AEs with onset or worsening on or after the first dose of study treatment, up to and including 28 days after the last dose.

\section{RESULTS}

\section{Baseline characteristics}

The study period was from the 24th October 2007 (first patient screened) to 3rd December 2008 (last patient completed the study). The flow of patients through the study is shown in Fig. 2. Baseline characteristics of the patient population (SES) are presented in Table 1.

This report presents data for the SES $(n=97)$, the FAS $(n=92)$ and the PPS $(n=81)$. Protocol deviations leading to exclusion from the FAS were identified in $5(5.2 \%)$ of the 97 treated patients and were mainly due to patients not reaching the maximum once-daily

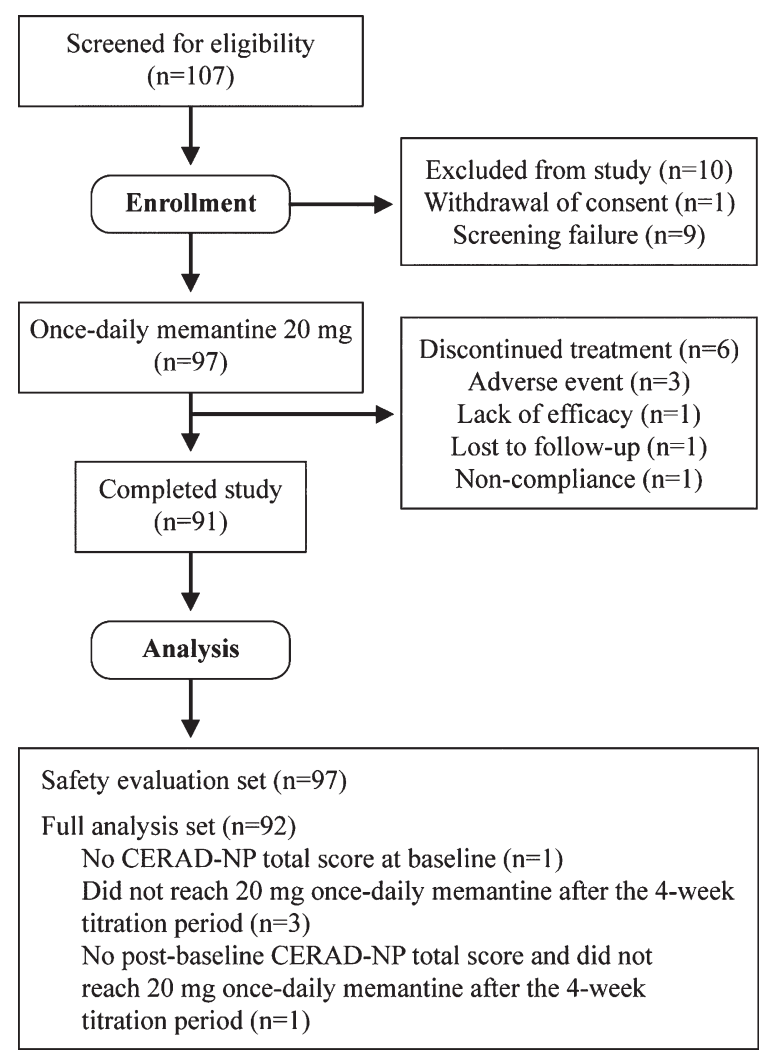

Fig. 2. Patient flow through study.
Table 1

Patient characteristics at baseline (SES)

\begin{tabular}{lc}
\hline Patient characteristic & $\begin{array}{c}\text { Study population } \\
(n=97)\end{array}$ \\
\hline Gender, female $n(\%)$ & $54(55.7)$ \\
Race, Caucasian $n(\%)$ & $97(100)$ \\
Mean age, years (SD) & $74.5(8.5)$ \\
Range & $53-91$ \\
Mean MMSE score ${ }^{\text {a }}(\mathrm{SD})$ & $16.0(3.7)$ \\
Range & $3-20$ \\
Mean duration of AD, years (SD) & $2.09(2.06)$ \\
Range & $0-8.7$ \\
Mean duration of education, years (SD) & $11.3(2.6)$ \\
Range & $8-18$ \\
\hline aMSE was measured at screening $(n=92)$. &
\end{tabular}

memantine dose of $20 \mathrm{mg}$ after the 4-week titration period (Fig. 2). Major protocol deviations leading to exclusion from the PPS were identified in $16(16.5 \%)$ of the 97 treated subjects. These consisted of the 5 subjects already excluded from the FAS above, 2 subjects who had prohibited concomitant medication and premature trial termination prior to visit 5,4 subjects who violated inclusion criteria ( 3 had MMSE $>19$ at screening), 4 subjects who had lack of compliance/reduced dose, and 1 subject for whom the CERAD-NP total score at visit 5 was missing. Overall, compliance with study medication was very good in the majority of patients with a percentage compliance (i.e., number of tablets taken calculated as a percentage of the number of tablets prescribed) of $95.8 \%$.

\section{Validation}

Correlations of the FLCI with sub-items of the CERAD-NP and verbal fluency testing gave satisfying evidence for construct validity (Table 2$)$. The FLCI total score correlated highest $(r=0.80)$ with the CERAD modified Boston Naming Test (BNT), a wellknown measure of naming and language ability.

The FLCI correlated moderately with the CERAD verbal fluency test $(r=0.54)$ and the phonemic verbal fluency test $(r=0.39)$. Probably these correlations were smaller than with the CERAD modified Boston Naming Test, because both verbal fluency tests do not only capture language ability, but also executive ability; i.e., they are a less straightforward measure of language than the BNT. Moderate correlations of the FLCI with CERAD Word list recall and CERAD Word list recognition are likely due to their memory component apart from their language component. 
Table 2

Correlation of FLCI total score with other language items

\begin{tabular}{|c|c|c|c|c|c|}
\hline FLCI total score & Week 4 & Week 8 & Week 12 & Week 16 & Mean \\
\hline \multicolumn{6}{|l|}{ Verbal fluency } \\
\hline $\begin{array}{l}\text { Pearson correlation coefficient } \\
p \text {-value }\end{array}$ & $\begin{array}{c}0.54 \\
<0.0001\end{array}$ & $\begin{array}{c}0.47 \\
<0.0001\end{array}$ & $\begin{array}{c}0.56 \\
<0.0001\end{array}$ & $\begin{array}{c}0.57 \\
<0.0001\end{array}$ & 0.54 \\
\hline \multicolumn{6}{|l|}{ Modified boston naming test } \\
\hline $\begin{array}{l}\text { Pearson correlation coefficient } \\
p \text {-value }\end{array}$ & $\begin{array}{c}0.77 \\
<0.0001\end{array}$ & $\begin{array}{c}0.80 \\
<0.0001\end{array}$ & $\begin{array}{c}0.80 \\
<0.0001\end{array}$ & $\begin{array}{c}0.82 \\
<0.0001\end{array}$ & 0.80 \\
\hline \multicolumn{6}{|l|}{ Phonemic verbal fluency } \\
\hline $\begin{array}{l}\text { Pearson correlation coefficient } \\
p \text {-value }\end{array}$ & $\begin{array}{l}0.29 \\
0.0055\end{array}$ & $\begin{array}{l}0.36 \\
0.0004\end{array}$ & $\begin{array}{c}0.44 \\
<0.0001\end{array}$ & $\begin{array}{l}0.45 \\
<0.0001\end{array}$ & 0.39 \\
\hline \multicolumn{6}{|l|}{ Word list recall } \\
\hline $\begin{array}{l}\text { Pearson correlation coefficient } \\
p \text {-value }\end{array}$ & $\begin{array}{l}0.26 \\
0.0126\end{array}$ & $\begin{array}{l}0.31 \\
0.0024\end{array}$ & $\begin{array}{l}0.27 \\
0.0085\end{array}$ & $\begin{array}{l}0.29 \\
0.0057\end{array}$ & 0.28 \\
\hline \multicolumn{6}{|l|}{ Word list recognition } \\
\hline $\begin{array}{l}\text { Pearson correlation coefficient } \\
p \text {-value }\end{array}$ & $\begin{array}{l}0.37 \\
0.0003\end{array}$ & $\begin{array}{l}0.30 \\
0.0032\end{array}$ & $\begin{array}{l}0.30 \\
0.0033\end{array}$ & $\begin{array}{c}0.40 \\
<0.0001\end{array}$ & 0.34 \\
\hline
\end{tabular}

\section{Effects}

The primary efficacy endpoint was the mean change from baseline to Week 12, in the CERAD-NP total score. The mean change from baseline in the CERADNP total score increased (improved) at each visit from baseline to Week 12 and, following cessation of treatment, decreased slightly at Week 16 (Fig. 3). The CERAD-NP total score was significantly greater after 12 weeks of once-daily memantine treatment compared with baseline $(5.9 \pm 8.8 ; p<0.0001)$ (Fig. 3$)$. The significant improvement in CERAD-NP total score over baseline was evident from Week 4 (3.3 \pm 6.1 ; $p<0.0001)$ and was maintained at Week 16 following 4 weeks of wash-out $(4.4 \pm 8.0 ; p<0.0001)$, despite a significant reduction in the mean change from Week 16 compared with that observed at Week $12(2.0 \pm 5.5$; $p=0.0004$ ) (Fig. 3). Results for the PPS were comparable to those of the FAS, confirming the statistically significant effect of once-daily memantine on the cognitive status of patients with $\mathrm{AD}$ as assessed by CERAD-NP total score. The results for the CERADNP single-item subscores of the sub-tests are presented in Table 3. For each single-item subscore, the results for the PPS were similar to the FAS results, with the exception of the two sub-tests for constructional ability: constructional praxis at Week 12 (FAS, $0.3 \pm 2.2$ $[p=0.0917]$; PPS, $0.5 \pm 2.0[p=0.0151])$ and figures recall at Week 4 (FAS, $0.4 \pm 2.2[p=0.0398]$; PPS, $0.4 \pm 2.3[p=0.0583])$.
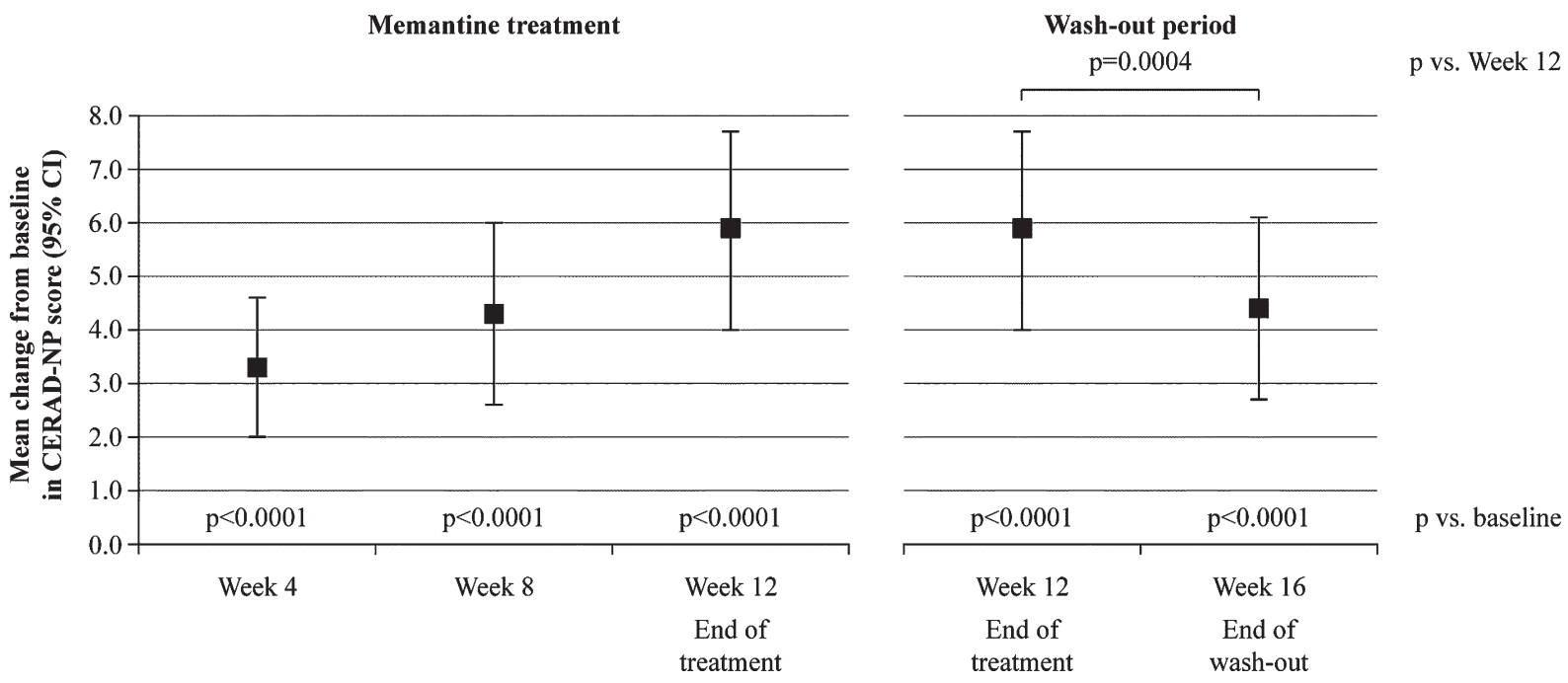

Fig. 3. Change from baseline over time in CERAD-NP total score (FAS). 
Table 3

Change from baseline over time in CERAD-NP single-item subscores, FLCI and ADCS-ADL 19 total scores, and in the Phonemic Verbal Fluency Test scores (FAS)

\begin{tabular}{|c|c|c|c|c|}
\hline & \multicolumn{4}{|c|}{ Memantine $20 \mathrm{mg} / \mathrm{day}$} \\
\hline & \multicolumn{4}{|c|}{ Change from baseline } \\
\hline & Week 4 & Week 8 & Week 12 & Week 16 \\
\hline \multicolumn{5}{|c|}{ CERAD-NP total score } \\
\hline$n$ & 92 & 92 & 92 & 87 \\
\hline Mean (SD) & $3.3(6.1)$ & $4.3(8.2)$ & $5.9(8.8)$ & $4.4(8.0)$ \\
\hline$p$-value & $<0.0001$ & $<0.0001$ & $<0.0001$ & $<0.0001$ \\
\hline \multicolumn{5}{|c|}{ CERAD-NP single-item } \\
\hline \multicolumn{5}{|c|}{ Verbal fluency (animal naming) } \\
\hline$n$ & 92 & 92 & 92 & 88 \\
\hline Mean (SD) & $0.6(2.6)$ & $1.0(2.8)$ & $0.8(3.0)$ & $0.8(2.9)$ \\
\hline$p$-value & 0.0200 & 0.0004 & 0.0065 & 0.0039 \\
\hline \multicolumn{5}{|c|}{ MBNT (modified Boston Naming Test) } \\
\hline$n$ & 92 & 92 & 92 & 88 \\
\hline Mean (SD) & $0.8(1.7)$ & $1.0(1.8)$ & $1.1(1.8)$ & $0.9(1.8)$ \\
\hline$p$-value & $<0.0001$ & $<0.0001$ & $<0.0001$ & $<0.0001$ \\
\hline \multicolumn{5}{|l|}{$M M S E$} \\
\hline$n$ & 91 & 92 & 92 & 88 \\
\hline Mean (SD) & $1.5(2.9)$ & $1.8(2.7)$ & $2.4(3.2)$ & $2.1(3.3)$ \\
\hline$p$-value & $<0.0001$ & $<0.0001$ & $<0.0001$ & $<0.0001$ \\
\hline \multicolumn{5}{|c|}{ Constructional ability } \\
\hline \multicolumn{5}{|c|}{ Constructional praxis } \\
\hline$n$ & 92 & 92 & 92 & 88 \\
\hline Mean (SD) & $0.2(1.8)$ & $0.2(2.1)$ & $0.3(2.2)$ & $-0.20(2.1)$ \\
\hline$p$-value & 0.1044 & 0.2477 & 0.0917 & 0.1965 \\
\hline \multicolumn{5}{|l|}{ Figures recall } \\
\hline$n$ & 91 & 91 & 91 & 87 \\
\hline Mean (SD) & $0.4(2.2)$ & $0.7(2.4)$ & $0.9(2.4)$ & $0.5(2.3)$ \\
\hline$p$-value & 0.0398 & 0.0025 & 0.0002 & 0.0258 \\
\hline \multicolumn{5}{|c|}{ Word list memory } \\
\hline \multicolumn{5}{|c|}{ Word list learning } \\
\hline$n$ & 92 & 92 & 92 & 87 \\
\hline Mean (SD) & $1.1(2.6)$ & $1.2(3.2)$ & $1.9(3.2)$ & $1.4(3.5)$ \\
\hline$p$-value & $<0.0001$ & 0.0002 & $<0.0001$ & 0.0002 \\
\hline \multicolumn{5}{|l|}{ Word list recall } \\
\hline$n$ & 92 & 92 & 92 & 87 \\
\hline Mean (SD) & $0.3(1.2)$ & $0.4(1.0)$ & $0.5(1.3)$ & $0.4(1.5)$ \\
\hline$p$-value & 0.0052 & 0.0006 & 0.0002 & 0.0083 \\
\hline \multicolumn{5}{|c|}{ Word list recognition - discriminability } \\
\hline$n$ & 92 & 92 & 92 & 87 \\
\hline Mean (SD) & $0.2(2.5)$ & $0.5(3.2)$ & $1.3(3.3)$ & $1.0(3.3)$ \\
\hline$p$-value & 0.2042 & 0.0625 & 0.0001 & 0.0021 \\
\hline \multicolumn{5}{|l|}{ FLCI } \\
\hline$n$ & 92 & 92 & 92 & 88 \\
\hline Mean (SD) & $2.9(5.4)$ & $3.5(6.8)$ & $4.4(6.8)$ & $3.6(7.0)$ \\
\hline$p$-value & $<0.0001$ & $<0.0001$ & $<0.0001$ & $<0.0001$ \\
\hline \multicolumn{5}{|l|}{$A D C S-A D L_{19}$} \\
\hline$n$ & 92 & 92 & 92 & 88 \\
\hline Mean (SD) & $0.7(3.0)$ & $0.6(4.4)$ & $0.7(5.0)$ & $0.1(5.5)$ \\
\hline$p$-value & 0.0184 & 0.1128 & 0.1026 & 0.4459 \\
\hline \multicolumn{5}{|c|}{ Phonemic Verbal Fluency Test (S-words) } \\
\hline$n$ & 92 & 92 & 92 & 88 \\
\hline Mean (SD) & $0.9(2.9)$ & $0.6(2.7)$ & $1.2(2.8)$ & $0.9(3.2)$ \\
\hline$p$-value & 0.0020 & 0.0234 & $<0.0001$ & 0.0036 \\
\hline
\end{tabular}

Statistical analysis for the secondary efficacy endpoints was performed post-hoc using a one-sided, single-group $t$-test at a significance level of $2.5 \%$. 

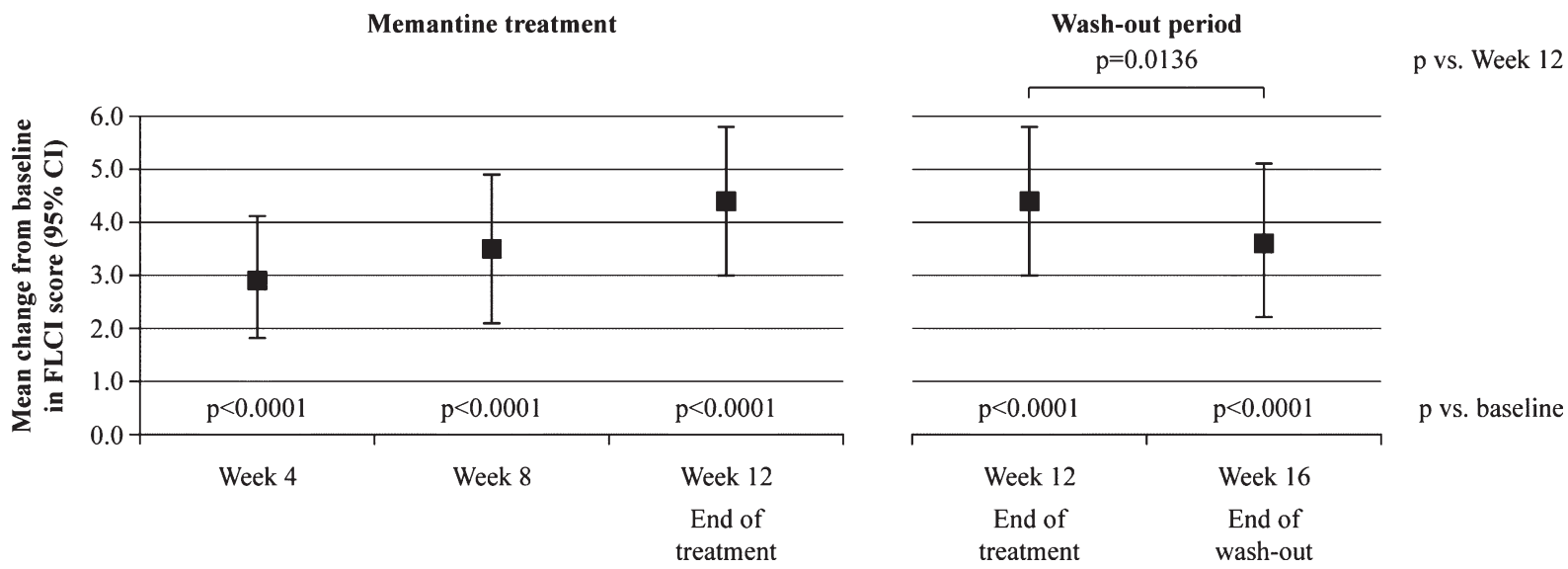

Fig. 4. Change from baseline over time in FLCI total score (FAS).
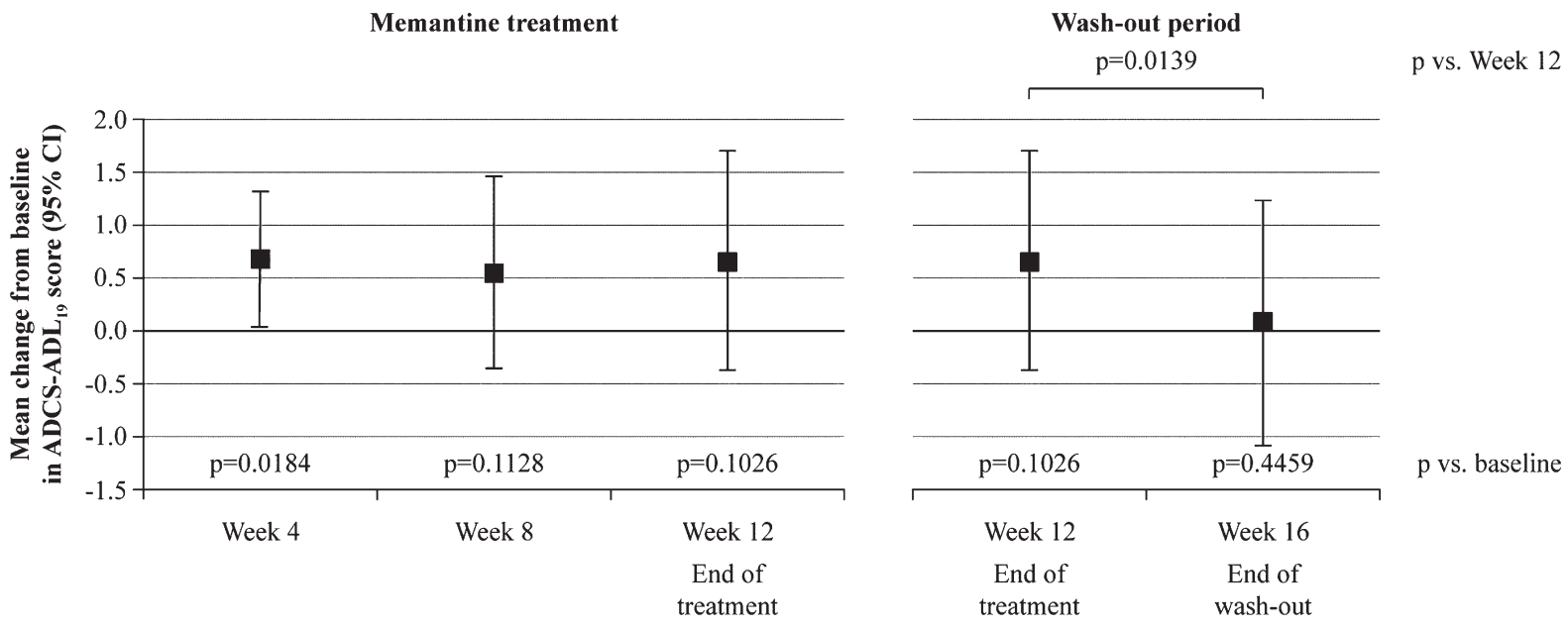

Fig. 5. Change from baseline over time in $\mathrm{ADCS}-\mathrm{ADL}_{19}$ total score (FAS).

The secondary efficacy variable, FLCI total score, showed a steady increase (improvement) in the mean change from baseline at each visit from baseline to Week 12, decreasing slightly at Week 16 (Fig. 4; Table 3). After 12 weeks of once-daily memantine treatment, the mean change from baseline in FLCI total score was significantly greater compared with baseline $(4.4 \pm 6.8 ; p<0.0001)$ (Fig. 4; Table 3). The significant improvement in FLCI total score over baseline was evident from Week $4(2.9 \pm 5.4 ; p<0.0001)$ and was maintained at Week 16 following 4 weeks of wash-out $(3.6 \pm 7.0 ; p<0.0001)$, despite a significant reduction in the mean change from Week 16 compared with that observed at Week $12(1.1 \pm 4.8 ; p=0.0136)$ (Fig. 4; Table 3).
Analysis of the secondary efficacy endpoint, mean

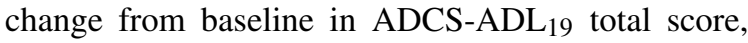
showed only slight post-baseline improvements from baseline to Week 12, returning to near baseline values at Week 16 (Fig. 5; Table 3). Following 4 weeks of wash-out, the mean change from baseline in ADCS$\mathrm{ADL}_{19}$ total score had significantly decreased at Week 16 compared with that observed at Week $12(0.9 \pm 3.7$; $p=0.0139$ ) (Fig. 5).

The results for the secondary efficacy endpoint assessing the response versus baseline in CGI-C over time indicated that the majority of patients experienced an improvement or stabilization of the disease from as early as Week 4 (Fig. 6). After 4 weeks of washout, a higher number of patients showed some degree 


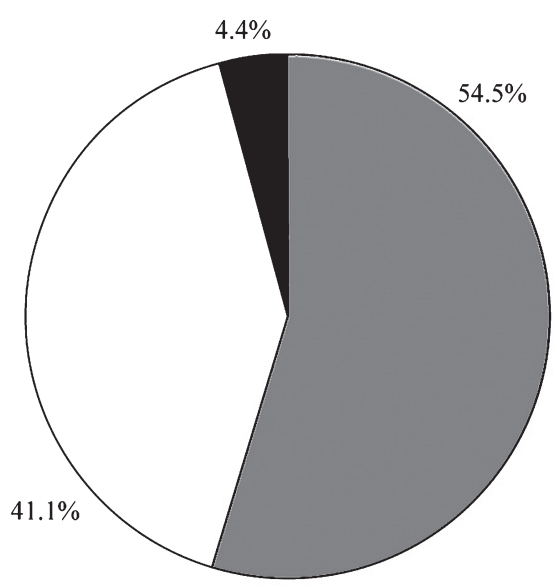

Week 4

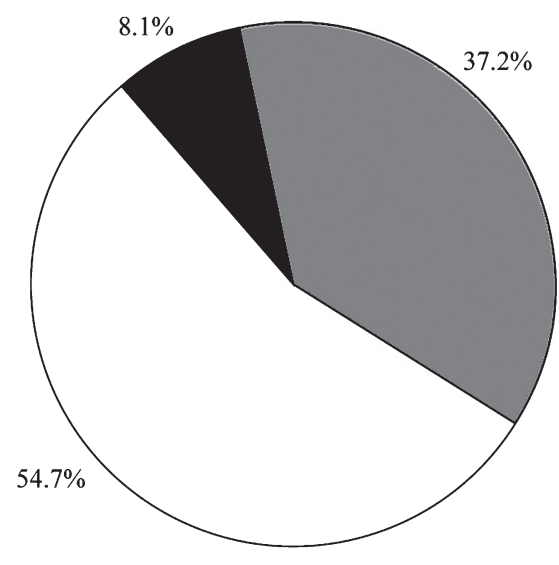

Week 12

(end of treatment)

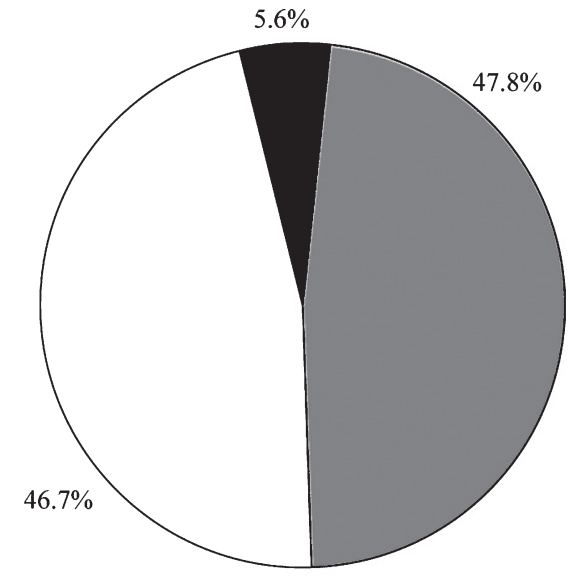

Week 8

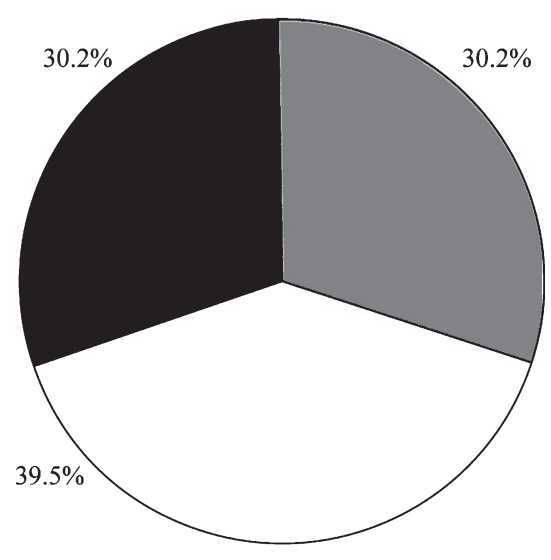

Week 16

(wash-out period)

Improved $\square$ Stabilised $\square$ Declined

Fig. 6. Change from baseline over time in CGI-C (FAS). Improved = very much improved, much improved, minimally improved; no change = no change; worsened = very much worse, much worse, minimally worse; data shown as percentage in each group.

of deterioration at Week 16, compared with all other time points (Fig. 6). The results for the Phonemic Verbal Fluency Test showed a significant improvement over baseline after 12 weeks of once-daily memantine treatment $(1.2 \pm 2.8 ; p<0.0001)$.

For each of the secondary endpoints, the results in the PPS were similar to the FAS results.

The results of the subgroup analysis defined by MMSE score at screening for the primary efficacy endpoint, CERAD-NP total score are presented in Table 4. In both subgroups, there was a significant increase (improvement) in CERAD-NP total score at Week 12 compared to baseline (Table 4). After 4 weeks of washout, patients with an MMSE $<15$ at screening showed a decrease (deterioration) in CERAD-NP total score compared to Week 12, which was not significantly different from baseline. In contrast, patients with MMSE $\geq 15$ at screening showed CERAD-NP total scores that were decreased compared to Week 12, but still significantly increased compared to baseline (Table 4). For the post-hoc MMSE subgroup analysis, the results for the PPS were similar to the FAS, with the exception of the Week 8 scores in the MMSE $<15$ subgroup (FAS, $2.3 \pm 5.9$ [ $p=0.0430]$; PPS, $2.4 \pm 6.2[p=0.0612]$ ).

Most, but not all of the ten FLCI subtests had significant changes from baseline to week 12 (end of study medication, Table 5). The most robust changes were found for "greeting and naming", "pantomime", "word reading and comprehension", "gesture" and "conversation". Probably, lack of range was the most important 
Table 4

Change from baseline over time in CERAD-NP total score for the post-hoc subgroup analysis defined by MMSE score at screening MMSE $<15$ or MMSE $\geq 15$ (FAS)

\begin{tabular}{lcccc}
\hline & \multicolumn{4}{c}{ Memantine 20 mg/day } \\
\cline { 2 - 5 } & Week 4 & Week 8 & Week 12 & Week 16 \\
\cline { 2 - 5 } & & & & \\
\hline MMSE $<15$ & 21 & 21 & 21 & 19 \\
$n$ & $3.3(5.5)$ & $2.3(5.9)$ & $4.1(7.6)$ & $2.7(8.4)$ \\
Mean (SD) & 0.0062 & 0.0430 & 0.0104 & 0.0892 \\
$p$-value & & & & \\
MMSE $\geq 15$ & 71 & 71 & 71 & 68 \\
$n$ & $3.3(6.4)$ & $4.8(8.7)$ & $6.4(9.1)$ & $4.8(7.9)$ \\
Mean (SD) & $<0.0001$ & $<0.0001$ & $<0.0001$ & $<0.0001$ \\
$p$-value & &
\end{tabular}

Statistical analysis for the secondary efficacy endpoints was performed post-hoc using a one-sided, single-group $t$-test at a significance level of $2.5 \%$.

reason in subtest "following commands", and bottom effects influenced "comprehension of signs" and "reminiscing".

\section{Safety and tolerability}

The mean exposure of patients to once-daily memantine was $83.7 \pm 9.2$ days with a cumulative exposure of 8,116 days.

During the course of the study, $38(39.2 \%)$ patients experienced TEAEs. The most frequent TEAEs, occurring in $\geq 2$ patients, are presented in Table 6 . Fatigue $(n=6 ; 6.2 \%)$ was the most commonly reported TEAE among patients, followed by anorexia, nasopharyngitis, and nausea each occurring in $4(4.1 \%)$ patients (Table 5). The majority of TEAEs were mild or moderate in intensity. TEAEs were considered by the investigator to be related to study medication in 13 $(13.4 \%)$ patients. The related TEAEs that occurred in $\geq 1$ patient were fatigue $(n=4 ; 4.1 \%)$, nausea $(n=3$; $3.1 \%)$, anorexia $(n=3 ; 3.1 \%)$, and agitation $(n=2$; $2.1 \%)$.

Three $(3.1 \%)$ patients experienced a TEAE that led to premature study termination, reported as hypertensive emergency $(n=1 ; 1.0 \%)$, diarrhea $(n=1 ; 1.0 \%)$, and chills, pyrexia and confusional state $(n=1 ; 1.0 \%)$; only the event of diarrhea was considered by the investigator to be related to study medication.

Treatment-emergent serious AEs (TESAEs) were reported for $3(3.1 \%)$ patients: syncope $(n=1 ; 1.0 \%)$, dehydration resulting from an insufficient intake of fluids (exsiccosis) due to the common cold $(n=1 ; 1.0 \%)$, and acute myeloid leukemia $(n=1 ; 1.0 \%)$. None of these events were considered by the investigator to be related to study medication.
One $(1.0 \%)$ death occurred during the study due to acute myeloid leukemia; the event was not considered by the investigator to be related to study medication.

Analysis of clinical laboratory data and vital signs raised no safety concerns.

\section{DISCUSSION}

The study evaluated a once-daily regimen of memantine for efficacy, safety, and tolerability, in the treatment of AD-related cognition and communication issues in patients with moderate to severe AD.

The results of this study showed that after 12 weeks of treatment with once-daily memantine, patients with moderate to severe AD significantly improved in cognition and functional communication, from as early as 4 weeks after the start of treatment. Results in the PPS were consistent with those in the FAS.

The benefits of memantine illustrated in this current study, support previous findings with memantine and other anti-dementia medications [12]. Indeed, recent clinical studies have suggested benefits for communication with the use of the AChEI, rivastigmine, in terms of spontaneous speech in patients with mild to moderate $\mathrm{AD}$ following 6 months of treatment [4]. There is also evidence to suggest that the $N$ methyl- $D$-aspartate receptor antagonist, memantine, may improve communication in patients with $\mathrm{AD}$. In a retrospective analysis of a randomized, controlled trial [13], memantine showed significant benefits over placebo in the Alzheimer's Disease Assessment Scalecognitive subscale (ADAS-Cog) individual item of language, following 24 weeks of treatment in patients with mild to moderate AD [14]. Similar findings have been reported for patients with moderate to severe AD following 24 weeks of memantine treatment by way of two post-hoc analyses of pooled data $[15,16]$. In the first analysis, memantine treatment resulted in significantly more patients improving on a language cluster than placebo (assessed using the ADAS-cog and the Severe Impairment Battery; SIB) [15]. In the second analysis, memantine showed significant benefits over placebo in terms of single items such as commands, orientation, comprehension, remembering test instructions, and language (assessed using the ADAS-Cog and SIB) [16].

As the present study was open-label, there was the potential for bias due to both patients and caregivers, as well as raters, being aware of the treatment schedules, i.e., when memantine was started and stopped. However, the total scores for CERAD-NP and FLCI 
Table 5

FLCI single item analysis

\begin{tabular}{|c|c|c|c|c|c|}
\hline \multirow[t]{3}{*}{ FLCI } & \multicolumn{5}{|c|}{ Memantine $20 \mathrm{mg} /$ day } \\
\hline & \multicolumn{5}{|c|}{ Change from baseline } \\
\hline & Baseline value & Week 4 & Week 8 & Week 12 & Week 16 \\
\hline \multicolumn{6}{|c|}{ Greeting and naming $(0-15)$} \\
\hline$n$ & 92 & 92 & 92 & 92 & 88 \\
\hline Mean (SD) & $10.7(2.6)$ & $0.6(1.2)$ & $1.0(1.6)$ & $1.3(1.7)$ & $1.1(1.6)$ \\
\hline$p$-value & & $<0.0001$ & $<0.0001$ & $<0.0001$ & $<0.0001$ \\
\hline \multicolumn{6}{|c|}{ Answering questions $(0-12)$} \\
\hline$n$ & 92 & 92 & 92 & 92 & 88 \\
\hline Mean (SD) & $9.3(2.8)$ & $0.4(1.6)$ & $0.5(2.1)$ & $0.6(2.5)$ & $0.6(2.3)$ \\
\hline$p$-value & & 0.0121 & 0.0062 & 0.0127 & 0.0125 \\
\hline \multicolumn{6}{|l|}{ Writing (0-11) } \\
\hline$n$ & 92 & 92 & 92 & 92 & 88 \\
\hline Mean (SD) & $6.6(3.6)$ & $0.5(2.0)$ & $0.4(2.0)$ & $0.4(2.0)$ & $0.5(2.1)$ \\
\hline$p$-value & & 0.0138 & 0.0180 & 0.0180 & 0.0163 \\
\hline \multicolumn{6}{|c|}{ Comprehension of signs $(0-6)$} \\
\hline$n$ & 92 & 92 & 92 & 92 & 88 \\
\hline Mean (SD) & $5.6(1.1)$ & $0.1(0.6)$ & $0.1(0.9)$ & $0.1(0.7)$ & $0.0(0.7)$ \\
\hline$p$-value & & 0.0313 & 0.2527 & 0.1896 & 0.3798 \\
\hline \multicolumn{6}{|c|}{ Word reading and comprehension $(0-18)$} \\
\hline$n$ & 92 & 92 & 92 & 92 & 88 \\
\hline Mean (SD) & $15.5(3.3)$ & $0.3(1.5)$ & $0.3(2.1)$ & $0.5(2.0)$ & $0.5(2.3)$ \\
\hline$p$-value & & 0.0175 & 0.0803 & 0.0049 & 0.0292 \\
\hline \multicolumn{6}{|c|}{ Reminiscing (0-6) } \\
\hline$n$ & 92 & 92 & 92 & 92 & 88 \\
\hline Mean (SD) & $3.7(1.6)$ & $0.1(1.6)$ & $0.1(1.6)$ & $0.2(1.7)$ & $0.1(1.7)$ \\
\hline$p$-value & & 0.3252 & 0.2572 & 0.0778 & 0.3092 \\
\hline \multicolumn{6}{|c|}{ Following commands $(0-2)$} \\
\hline$n$ & 92 & 92 & 92 & 92 & 88 \\
\hline Mean (SD) & $1.7(0.5)$ & $0.0(0.4)$ & $0.0(0.6)$ & $0.0(0.6)$ & $0.0(0.5)$ \\
\hline$p$-value & & 0.4667 & 0.2901 & 0.2479 & 0.4214 \\
\hline \multicolumn{6}{|c|}{ Pantomime (0-9) } \\
\hline$n$ & 92 & 92 & 92 & 92 & 88 \\
\hline Mean (SD) & $5.9(2.5)$ & $0.4(1.4)$ & $0.6(1.8)$ & $0.5(1.7)$ & $0.4(1.8)$ \\
\hline$p$-value & & 0.0021 & 0.0011 & 0.0013 & 0.0153 \\
\hline \multicolumn{6}{|l|}{ Gesture (0-4) } \\
\hline$n$ & 92 & 92 & 92 & 92 & 88 \\
\hline Mean (SD) & $3.3(0.9)$ & $0.2(0.8)$ & $0.2(0.9)$ & $0.2(0.8)$ & $0.1(0.8)$ \\
\hline$p$-value & & 0.0042 & 0.0416 & 0.0014 & 0.0370 \\
\hline \multicolumn{6}{|c|}{ Conversation $(0-4)$} \\
\hline$n$ & 92 & 92 & 92 & 92 & 88 \\
\hline Mean (SD) & $3.2(1.1)$ & $0.2(0.9)$ & $0.3(0.9)$ & $0.3(1.1)$ & $0.2(1.1)$ \\
\hline$p$-value & & 0.0087 & 0.0041 & 0.0011 & 0.0229 \\
\hline
\end{tabular}

Numbers in parenthesis show range of scores. Mean values represent change from baseline.

remained significantly higher than baseline following the period of wash-out despite the fact that patients and caregivers knew that memantine was no longer being given.

It is important to note that although there was a significant reduction in the cognition and functional communication scores at the end of the wash-out period (Week 16) compared with the end of treatment (Week 12), the respective scores were still significantly higher after 4 weeks of wash-out than they were at baseline. The sustained positive effects of memantine at 4 weeks after wash-out are unlikely to be explained by the activity of the drug itself, because the esti- mated elimination half-life of memantine is $60-100 \mathrm{~h}$ [17]. It is an interesting hypothesis that memantine induces improvement of neuronal activity, that results in better synaptic function and ultimately neuronal plasticity, with benefits that persist after cessation of therapy. Similar effects have been discussed after wash-out of levodopa in patients with Parkinson's disease [18]. In their study, based on presumed toxic effects of levodopa on dopaminergic neurones, reduced performance in the levodopa-treated group relative to placebo-treated patients was expected following washout [18]. However, patients that had received levodopa for 40 weeks still showed benefits after a wash-out 
Table 6

Treatment-emergent adverse events occurring in $\geq 2$ patients (SES)

\begin{tabular}{lc}
\hline $\begin{array}{l}\text { Treatment-emergent adverse event } \\
\text { (preferred term) }\end{array}$ & Number of patients $(\%)$ \\
\hline Patients with $\geq 1$ TEAE & $38(39.2)$ \\
Fatigue & $6(6.2)$ \\
Anorexia & $4(4.1)$ \\
Nasopharyngitis & $4(4.1)$ \\
Nausea & $4(4.1)$ \\
Agitation & $2(2.1)$ \\
Arthralgia & $2(2.1)$ \\
Confusional state & $2(2.1)$ \\
Delirium & $2(2.1)$ \\
Depression & $2(2.1)$ \\
Influenza & $2(2.1)$ \\
Rhinitis & $2(2.1)$ \\
Syncope & $2(2.1)$ \\
Vertigo & $2(2.1)$ \\
\hline
\end{tabular}

$\mathrm{TEAE}=$ treatment-emergent adverse event.

period of 2 weeks compared with the placebo-treated group [18]. The observed reduction of benefit upon withdrawal of treatment highlights the need for patients to be treated continuously in order to gain most benefit.

In our study we did not observe a discontinuation syndrome [19] during the wash-out period after cessation of memantine. This has recently been described in two patients after abrupt cessation of memantine [20]. Similarly, a discontinuation syndrome was reported after cessation of donepezil [21]. In contrast to the sustained effects after a 4-week wash out of memantine, no further positive effects are observed after a 24-week treatment period with donepezil followed by a 6-week wash out period [22].

Memantine also demonstrated a stabilization of symptoms over the 12-week treatment period in terms of ADL and CGI-C, indicating that memantine allows patients to retain their everyday functioning by providing an overall beneficial response to treatment.

Once-daily memantine showed a favorable safety and tolerability profile. All the TEAEs reported were to be expected in $\mathrm{AD}$ patients receiving memantine. The most commonly reported TEAE was fatigue which is a known adverse reaction to memantine. Anorexia, agitation, confusional state, delirium, depression, and vertigo have all been associated with $A D$. Nausea and syncope are known side effects of AChEI, which were a common concomitant medication in the present study. Furthermore, reports of nasopharyngitis, arthralgia, influenza, and rhinitis are not unexpected in an elderly population such as the patients enrolled in this study. The one death that occurred during the study was not considered to be related to treatment and raised no safety concerns for the once-daily regimen of memantine.
In conclusion, the study demonstrated that in patients with moderate to severe $\mathrm{AD}$ once-daily memantine improved cognition and functional communication and that treatment was safe and well tolerated.

In the present study, we used and validated the German language version of the FLCI for the first time. Furthermore, we demonstrated that the FLCI and the BNT are sensitive to measure improvement of language and communication deficits during treatment with memantine. We suggest to not only test $\mathrm{AD}$ patients for cognitive function but also for their language capacity.

The extent of the sustained effects of memantine as observed in the CERAD-NP, the FLCI, and CGI-C are surprising. This would argue against a predominant placebo effect in the results observed during the first 12 weeks. It is unlikely that these findings are the result of repeated testing with an interval of 4 weeks, because the patients included were already moderately to severely impaired. Furthermore, the CERAD-NP is a complex test battery that consists of different items and is, therefore, not dependent on performance in a single item. In most cases, the item scores reflected the results for the total score, i.e., steady improvement over time, tailing off following wash-out to values that were similar to the improvement seen at Week 8. In addition, similar effects were seen in two language and communication tests.

The conclusions drawn from this study should be interpreted cautiously, due to the open label design. However, a similarly designed placebo-controlled study confirmed our findings [23].

\section{ACKNOWLEDGMENTS}

The study sponsor was Merz Pharmaceuticals $\mathrm{GmbH}$, Eckenheimer Landstr. 100, 60318 Frankfurt/Main, Germany. The authors would like to thank all the study Principal Investigators from all the active sites who enrolled patients, as follows:

\section{Principal Investigators at active sites:}

M. Rainer, Wien; F. Leblhuber, Linz; H. Schubert, Hall/Tirol; C.-W. Wallesch, Magdeburg; J. Schulz, Göttingen; K. Hager, Hannover; J. Pantel, Frankfurt am Main; U. Siedl, Heidelberg; C. von Arnim, Ulm; K. Bürger, München; A. Kurz, München; H.H. Klünemann, Regensburg; H.-J. Gertz, Leipzig; R. Ihl, Krefeld; P. Kalus, Berlin; L.M. Drach, Schwerin; 
J. Peltz, Hattingen; G. Hamann, Wiesbaden; H. Jahn, Hamburg; G. Schumann, Bochum

Jörg B. Schulz has received research support from the BMBF and the DFG. Alexander Kurz has received research support from BMBF, BMG, and DFG.

Authors' disclosures available online (http://www.jalz.com/disclosures/view.php?id=785).

\section{REFERENCES}

[1] Bullock R, Hammond G (2003) Realistic expectations: the management of severe Alzheimer disease. Alzheimer Dis Assoc Disord 17(Suppl 3), S80-S85.

[2] Cummings JL (2004) Alzheimer's disease. N Engl J Med 351, 56-67.

[3] Georges J, Jansen S, Jackson J, Meyrieux A, Sadowska A, Selmes M (2008) Alzheimer's disease in real life - the dementia carer's survey. Int J Geriatr Psychiatry 23, 546-551.

[4] Visch Brink EG, van Rhee Temme W, Rietveld T, Krulder JW, Van Harskamp F, van der Cammen TJ (2009) Improvement of spontaneous speech in early stage Alzheimer's with rivastigmine. J Nutr Health Aging 13, 34-38.

[5] Chandler MJ, Lacritz LH, Hynan LS, Barnard HD, Allen G, Deschner M, Weiner MF, Cullum CM (2005) A total score for the CERAD neuropsychological battery. Neurology $\mathbf{6 5}$, 102-106.

[6] Morris JC, Heyman A, Mohs RC, Hughes JP, van Belle G, Fillenbaum G, Mellits ED, Clark C (1989) The Consortium to Establish a Registry for Alzheimer's Disease (CERAD). Part I. Clinical and neuropsychological assessment of Alzheimer's disease. Neurology 39, 1159-1165.

[7] Bayles K, Tomoeda C (1994) The Functional Linguistic Communication Inventory. Canyonlands Publishing, Inc.

[8] Galasko D, Bennett D, Sano M, Ernesto C, Thomas R, Grundman M, Ferris S (1997) An inventory to assess activities of daily living for clinical trials in Alzheimer's disease. The Alzheimer's Disease Cooperative Study. Alzheimer Dis Assoc Disord 11(Suppl 2), S33-S39.

[9] Galasko D, Schmitt F, Thomas R, Jin S, Bennett D; Alzheimer's Disease Cooperative Study (2005) Detailed assessment of activities of daily living in moderate to severe Alzheimer's disease. J Int Neuropsychol Soc 11, 446-453.

[10] Schneider LS, Olin JT, Doody RS, Clark CM, Morris JC, Reisberg B, Schmitt FA, Grundman M, Thomas RG, Ferris SH (1997) Validity and reliability of the Alzheimer's disease Cooperative Study Clinical Global Impression of Change. The Alzheimer's Disease - Cooperative Study. Alzheimer Dis Assoc Disord 11(Suppl 2), S22-S32.
[11] Troyer AK (2000) Normative data for clustering and switching on verbal fluency tasks. J Clin Exp Neuropsychol 22, 370-378.

[12] Tariot PN, Farlow MR, Grossberg GT, Graham SM, McDonald S, Gergel I; Memantine Study Group (2004) Memantine treatment in patients with moderate to severe Alzheimer disease already receiving donepezil: a randomized controlled trial. JAMA 291, 317-324.

[13] Peskind ER, Potkin SG, Pomara N, Ott BR, Graham SM, Olin JT, McDonald S (2006) Memantine treatment in mild to moderate Alzheimer disease: a 24-week randomized, controlled trial. Am J Geriatr Psychiatry 14, 704-715.

[14] Pomara N, Ott BR, Peskind E, Resnick EM (2007) Memantine treatment of cognitive symptoms in mild to moderate Alzheimer disease: secondary analyses from a placebocontrolled randomized trial. Alzheimer Dis Assoc Disord 21, 60-64.

[15] Emre M, Mecocci P, Stender K (2008) Pooled analyses on cognitive effects of memantine in patients with moderate to severe Alzheimer's disease. J Alzheimers Dis 14, 193-199.

[16] Mecocci P, Bladström A, Stender K (2009) Effects of memantine on cognition in patients with moderate to severe Alzheimer's disease: post-hoc analyses of ADAS-cog and SIB total and single-item scores from six randomized, doubleblind, placebo-controlled studies. Int J Geriatr Psychiatry 24, 532-538.

[17] Koch HJ, Szecsey A, Haen E (2004) NMDA-antagonism (memantine): an alternative pharmacological therapeutic principle in Alzheimer's and vascular dementia. Curr Pharm Des 10, 253-259.

[18] Fahn S, Oakes D, Shoulson I, Kieburtz K, Rudolph A, Lang A, Olanow CW, Tanner C, Marek K; Parkinson Study Group (2004) Levodopa and the progression of Parkinson's disease. N Engl J Med 351, 2498-2508.

[19] Gauthier S (2006) Managing discontinuation syndrome in patients with dementia. J Psychiatry Neurosci 31, 72.

[20] Kwak YT, Han I-W, Suk S-H, Koo M-S (2009) Two cases of discontinuation syndrome following cessation of memantine. Geriatr Gerontol Int 9, 203-205.

[21] Singh S, Dudley C (2003) Discontinuation syndrome following donepezil cessation. Int J Geriat Psychiatry 18, 282-284.

[22] Rogers SL, Farlow MR, Doody RS, Mohs R, Friedhoff LT (1998) A 24-week, double-blind, placebo-controlled trial of donepezil in patients with Alzheimer's disease. Donepezil Study Group. Neurology 50, 136-145.

[23] Hofbauer RK, Saxton J, Woodward M, Gilchrist N, Potocnik F, Yu SY, Hsu H-A, Graham SM, Perhach JL (2009) Effects of memantine on functional communication in patients with moderate AD: results of a 12-week placebo-controlled trial. Alzheimer's Dis Relat Disord 5(Suppl 1), Abst P1-Abst $\mathrm{P} 271$. 